\title{
Toward a framework for the natures of proportional reasoning in introductory physics
}

\author{
Andrew Boudreaux \\ Department of Physics and Astronomy, Western Washington University, 516 High St, Bellingham, WA 98225
}

Stephen E. Kanim

Department of Physics, New Mexico State University, 2850 Weddell St, Las Cruces, NM 88003

Alexis Olsho, Suzanne White Brahmia, and Charlotte Zimmerman

Department of Physics, University of Washington, Box 351560, Seattle, WA 98195-1560

Trevor I. Smith

Department of Physics \& Astronomy and Department of STEAM Education, Rowan University, 201 Mullica Hill Rd., Glassboro, NJ 08028

\begin{abstract}
We present a set of modes of reasoning about ratio and proportion as a means of operationalizing expert practice in physics. These modes, or natures of proportional reasoning, stem from consideration of how physicists reason in context and are informed by prior work in physics and mathematics education. We frame the natures as the core of an emerging framework for proportional reasoning in introductory physics, that will categorize the uses of proportional reasoning in introductory physics contexts, and provide guidance for the development of reliable assessments. We share results from preliminary assessment items indicating that university physics students have difficulty interpreting and applying ratios in context.
\end{abstract}




\section{INTRODUCTION}

Scientists and engineers think mathematically about events, or mathematize, when making sense of the world. In introductory physics, we would like our students to become more proficient mathematizers. Recent findings, however, suggest that students experience difficulty with many aspects of how mathematics is used in physics [1-4].

The use of ratios to characterize systems and processes is a hallmark of physics and a foundation for mathematizing. Introductory physics courses present new ratio quantities in rapid succession, such as velocity, acceleration, and coefficient of friction in mechanics, electric field, potential difference, and resistance in E\&M, heat capacity, frequency, and so on. Students not facile with ratio reasoning may struggle to employ these quantities for sense making, and instead may resort to memorization and pattern matching [5].

Students entering university physics will have practiced with ratios in pre-college math classes and likely worked with ratios in scientific contexts. Physics educators, however, have recognized the challenge that ratio reasoning poses to student success in physics. Indeed, Arnold Arons wrote in 1990 "One of the most severe gaps in the cognitive development of students is the failure to have mastered reasoning involving ratios...this disability is one of the most serious impediments to the study of science" [5]. Physics education researchers have identified some difficulties more systematically; for example, Trowbridge and McDermott found that "fewer than half of the students demonstrated sufficient qualitative understanding of acceleration as a ratio to be able to apply this concept in a real situation" [6].

We view proportional reasoning as complex and multifaceted. Patrick Thompson has described proportional reasoning as a broad set of interconnected and context dependent skills, represented by a "proportionality cloud" [7]. He has shown how the equivalent ratios paradigm - the narrow skill from school mathematics involving "this is to this as that is to that" - overlaps with a variety of topics typically treated as separate. We believe that developing such interconnected skills in the context of explaining realworld phenomena is central to "thinking like a physicist." More specifically, we view expert ability as involving intentional use of distinct modes of ratio reasoning and fluent shifting from one mode to another.

In this paper, we begin to unpack proportional reasoning from the perspective of an expert, by articulating specific modes of reasoning that arise in introductory physics. We refer to such modes as "natures," following our earlier work on the natures of negativity in physics [8]. ("Natures of negativity" refers to the ways physics experts reason about signs and signed quantities.) Although the natures of proportional reasoning presented here are preliminary, and not yet comprehensive, we believe they can form the core of a more complete set, which could then guide research on student reasoning and the design of assessment tools. A mature set of natures of proportional reasoning would in this way support systematic improvement of instruction.

\section{THEORETICAL FRAMEWORK}

We adopt the view that expert practice in physics involves coordinated procedural and conceptual mastery of the relevant mathematics. In articulating natures of proportional reasoning, we have thus avoided focus on computation alone. Gray and Tall have defined proceptual understanding, in which computational and conceptual facility coexist [9]. We have found this notion useful for examining the ways that experts reason with ratios in introductory physics contexts.

As an example, a proceptual understanding of fraction would entail fluid transition between the procedure of dividing 6 by 8 , and the conceptualization of $6 / 8$ as a precise quantification of portion. Similarly, a physics student with a proceptual understanding of acceleration would move between the computational procedure (dividing $\Delta \mathrm{v}$ by $\Delta \mathrm{t}$ ) and the conceptualization of the ratio as a quantity unto itself, which conveys the change in velocity corresponding to each unit of the elapsed time, and has its own units, properties, and utility. We regard a proceptual understanding of ratios in physics as the successful blend of computational procedures and conceptual understanding - where conceptual understanding includes the physical meaning of a ratio - the way the quantity represents a physical quality.

\section{RESEARCH METHODS}

This paper presents natures of proportional reasoning in introductory physics - i.e., specific ways experts reason. We have conducted systematic research on student facility with these natures. We have employed a variety of questions to investigate student reasoning, some drawn from the research literature and others developed ourselves. The questions do not emphasize computational skill; most require neither a calculator nor significant mental math. Questions were designed to target a single nature of proportional reasoning. Some questions involve a forced choice (i.e., multiple choice format), while others are free response. In all cases, students were asked to explain their reasoning.

The questions developed during the investigation underwent repeated cycles of modification. Student responses to initial versions were used to guide modifications to improve not only the clarity, but also the effectiveness of the questions in providing insights into student thinking. It was often challenging to create questions that did not trigger a common student response of trying to apply an equivalent fractions template.

Questions were administered in writing in general education, algebra-based, and calculus-based introductory physics courses at Western Washington University (WWU), New Mexico State University, and Rutgers University. Such questions were given on course exams and as ungraded 
quizzes. More than 1500 student responses have been collected overall.

Questions were also administered in individual, onehour, think-aloud interviews conducted at WWU with over 20 student volunteers from general education and calculusbased introductory physics courses as well as an introductory physics course for preservice elementary teachers. A semistructured protocol was used: the interviewer posed specific proportional reasoning questions and asked the interview subject to "think out loud." The interviewer clarified the questions as needed, prompted the subject to explain their thinking after sustained periods of silence, and asked the subject to elaborate on brief or unclear statements. The interviewer did not, however, offer hints or ask guiding questions. The interviews were videotaped and transcribed.

Responses on forced choice questions have been analyzed to obtain correct response rates; these results serve as an indicator of the general level of student facility with specific modes of ratio reasoning. (However, discussion of variations in performance between student populations is beyond the scope of this paper.) Thematic analysis has been applied to written explanations and interview transcripts to identify common reasoning difficulties [10]. Section IV shares selected findings from both types of analysis.

\section{NATURES OF PROPORTIONAL REASONING IN INTRODUCTORY PHYSICS}

Here we present three natures of proportional reasoning in introductory physics, describing each and briefly sharing selected results of research on student facility. (We hope to share more details of our research findings in future publications.) In articulating the natures, we draw on prior work in physics and math education, as well as our own collective teaching experience. We view these natures as a foundation on which to build a more complete framework.

The first two natures - verbal interpretation of a ratio and construction of a ratio from constituent quantities - are closely related under the overarching construct of unit rate. The third nature - application of a ratio to determine an unknown amount - may in some sense represent a higher order skill, i.e., a mode of reasoning that is supported by the first two natures. Articulating specific natures helps to operationalize proportional reasoning ability and can guide the development of tools for assessing fluency.

These three natures, discussed in detail in sections A.1, A.2 and B below, are broadly consistent with the proportional reasoning learning targets identified in the Common Core State Standards for Mathematics [11]. For example, the standard "Understand the concept of unit rate $a / b$ associated with the ratio $a: b$ and use rate language in the context of a ratio relationship" aligns closely with verbal interpretation of ratios (see A.1). We do not claim that students consciously marshal these natures of reasoning when solving problems, or that the natures stem from isolated cognitive entities or form a coherent basis for modeling cognition. Rather, they are presented as a practical guide for assessment and the development of instruction.

\section{A. Natures related to unit rate}

Verbal interpretation of ratio and the construction of a ratio constitute complementary natures of proportional reasoning about unit rate. Indeed, these two reasoning modes are in some sense inverses of one another. Verbal interpretation of ratio requires students to "translate" a given ratio-based quantity into everyday language in order to express the specific information the quantity provides about a system or process. For example, if the speed of a certain uniform motion is known to be $50 \mathrm{mph}$, a student can interpret the number 50 to recognize that the object travels 50 mi for each hour of the motion. A general notion of equal sharing of two quantities (i.e., that equal increments of the elapsed time correspond to equal increases in the distance traveled) is here quantified in a specific way through "translation" of the technical term "per" as "for each."

Conversely, ratio construction asks students to use two constituent quantities to construct a mathematical expression that corresponds to a given verbal description. For example, if the student is told that a car travels 240 miles in a 6-hr trip, and is asked to determine how many miles the car travels in each hour of the motion, she could construct the ratio 240/6.

\section{Verbal interpretation of ratio}

We regard the verbal interpretation of ratios as a foundation for proportional reasoning in physics. Such interpretation involves an internalized conception of the physical meaning of a ratio quantity, separate from but complementary to the recall and application of the formal mathematical definition. For example, an expert not only knows that the heat capacity $C$ is computed from the ratio $\mathrm{Q} / \Delta \mathrm{T}$, but also conceptualizes $C$ as the heat transfer associated with a 1-degree temperature change. For an expert, the verbal interpretation is part of an associative network of ideas surrounding the ratio quantity, readily activated along with the formula or other representation. Furthermore, when confronted with a novel quantity, the expert looks for a relevant interpretation, actively seeking physical meaning. While physics experts move fluidly between mathematical definitions and physical interpretations, introductory students may instead resort to memorized patterns of manipulation $[12,13]$.

Arnold Arons has written extensively on verbal interpretation of ratios. He notes that even after relevant instruction, students may be unable to interpret density as the number of units of mass for each unit of volume, or acceleration as the change in velocity for each unit of the elapsed time, and in general may use "per" without understanding its meaning as "for every" [5]. In a study involving Nigerian high school students, Akatugba and Wallace found that students frequently relied on algorithmic methods they could not explain [14]. Simon and Blume point 
out that students presented with a ratio quantity often fail to recognize it as derived from measurement [15]. For example, when asked to interpret a road sign " $6 \%$ grade," students explained that truck drivers might assign this number to a hill in much the same way that a subjective, numerical rating is assigned to a river rapid by expert kayakers. The students did not seem to recognize that the grade refers to the number of feet of change in elevation for each 100 feet of distance along the road - or even that the numerical value does in fact have a specific meaning which can (and should) be sought.

We have used a variety of written questions to probe student ability to interpret ratios in physics contexts. On one such question, the Porsche question, students are told "On its website, Porsche states 'Maximum: $21 \mathrm{mph}$ per second' in the technical specifications for a particular model" and are asked to "Describe the information this statement provides about the car, and explain the meaning of the word 'per' in this situation." This task has been administered in written form in introductory calculus-based mechanics courses at multiple institutions. Many students had difficulty interpreting "per second" as "in each second." (We note that many students struggled to distinguish velocity and acceleration, but do not discuss such difficulties here.) Some students recognized that the term per signals a relationship between two quantities, but did not articulate the essential aspect of proportion: "The overall per second parameter . . . is a time interval in which the car completed its task or whatever is being tested. 'Per' specifically relates the car's performance to time in this situation." This response seems to identify 21 as a change that occurs over time, but not as the change that occurs in a unit time. The specific interpretation of the numerical value, that each second corresponds to a change in velocity of $21 \mathrm{mph}$, has not been explicitly articulated, and may not have been internalized.

We have also administered questions that ask students to interpret non-standard ratios. For example, the Inverse $g$ question asks students to interpret the ratio quantity $0.1 \mathrm{~s}^{2} / \mathrm{m}$, used to describe the motion of a falling object. The quantity 0.1 is "non-standard" in that it is not a named quantity, and appears rarely if ever in physics text books. (Indeed, it is the inverse of the acceleration ratio featured in the Porsche question.) Although it does not have a common name, 0.1 can be interpreted as the number of seconds required for each $1 \mathrm{~m} / \mathrm{s}$ change in the falling object's velocity. We have posed this question in informal settings to physics faculty and graduate students. While they tend to express surprise or puzzlement initially, they generally arrive at a correct interpretation, often using units as a guide to their reasoning (i.e., experts often recognize that $0.1 \mathrm{~s}^{2} / \mathrm{m}$ can be recast as $0.1 \mathrm{~s}$ per $\mathrm{m} / \mathrm{s}$ ). In contrast, we have found the Inverse $\mathrm{g}$ question to be very challenging for introductory students, with only around $10 \%$ giving a correct interpretation at the end of a calculus-based mechanics course. While perhaps not surprising, this result suggests that the reasoning needed to interpret an unfamiliar ratio in context may not be readily accessible for many students.

\section{Construction of ratio}

We have administered a variety of tasks to probe the ability of students to construct a ratio that corresponds to a given verbal description. These tasks are, in a sense, the mirror image of the Porsche question presented above. Consider, for example, the Bobbing block question. This task involves a block suspended by a spring that bobs up and down in a repeating motion. Students are told that B bobs occur in 10 seconds, and are asked to write an expression for the number of seconds required for a single bob, and to explain their reasoning. We expected students to produce the expression 10/B. When given to over 500 students in multiple sections of general education introductory physics at WWU, and over 500 additional students in calculus-based mechanics at Rutgers University, between $45 \%$ to $60 \%$ of responses included the correct expression 10/B. Nearly all remaining responses gave the inverse of this expression (i.e., $\mathrm{B} / 10$ ). Thus, while most students recognized that the correct response involves a ratio (rather than a difference or product), many seemed to have difficulty constructing the ratio that matches the given verbal description.

One general education physics student who gave the B/10 response seemed to be answering a question different than the one asked: "The number of bobs in 10 sec is $B, B$ divided by 10 would give you bobs per a second." Rather than the period, this response is consistent with the frequency of the periodic motion. Another student explicitly acknowledged the period as the desired quantity, but still arrived at the incorrect answer B/10: "To find the time it takes for a bob we have to divide the number of bobs B by 10 seconds."

To explore student thinking in more depth, we have used tasks like the Bobbing block question in individual, thinkaloud interviews. In this setting, which allows for follow up questions to probe whether difficulties are surface level, associated with careful reading of text and interpretation of wording, or deeper level, associated with underlying reasoning, we have found that some students struggle in a sustained way to distinguish the expressions B/10 and 10/B, despite repeated prompts to ensure that the question was being interpreted as intended. In the interviews, some students exhibited an intuitive tendency to focus on rates of change with respect to time, rather than the alternative ratio construction (i.e., the time required for one repetition).

We have administered a variety of additional tasks that require students to construct a ratio from given measurements in order to match a desired interpretation. Results are similar to those from the Bobbing block question: many students provide the inverse of the expected ratio. Questions involving an unfamiliar target ratio (i.e., the volume of a homogeneous material required to make one unit of mass) tend to pose greater difficulty than more familiar ratios (the mass for each unit of volume). Although more prevalent among general education physics students, even calculus-based students exhibit difficulties of this type. 


\section{B. Application of ratio}

A ratio that characterizes a system or process allows quantitative predictions to be made about cases not yet observed. For example, the value of electrical resistance expresses an invariant relationship between changes in the current and voltage for a linear resistor - in which a quantity of Ohms is the number of volts needed for each amp of current. Knowledge of the resistance then allows a prediction of the current that will result from any applied voltage.

Applying a known ratio in this way involves essentially the same procedural skill as constructing a ratio: the equation $\mathrm{I}=\mathrm{V} / \mathrm{R}$ can be used to find the current from a measured voltage, or can be rearranged to find the resistance, given measurements of the voltage and current. Here, however, we treat application and construction of ratio as separate natures of proportional reasoning. Indeed, the form of quantitative reasoning that underlies applying a ratio is distinct from that underlying constructing a ratio. When dividing two quantities to form a ratio, we can imagine splitting the numerator quantity into equal-sized pieces, a rationale referred to as partitive division. When division is used to apply a ratio, however, one seeks to segment some total in units of size specified by the ratio, a conceptualization referred to as quotative division [16].

The application nature of proportional reasoning is ubiquitous in introductory physics; examples include the use of density to find mass and the use of velocity to find displacement. To examine student ability to apply ratios, we have developed tasks that differ in contextual features, but can all be solved with quotative reasoning. Two such tasks are shown in Fig. 1. The Traxolene question (Fig. 1.a) involves a typical introductory physics context, but a nonstandard ratio (i.e., $\mathrm{cm}^{3} / \mathrm{g}$ rather $\mathrm{g} / \mathrm{cm}^{3}$ ). The Olive oil question involves an "everyday context." When administered to over 500 students in calculus-based mechanics at Rutgers University, correct response rates were comparable: $66 \%$ on Traxolene and $61 \%$ on Olive oil. These results suggest that some university physics students have difficulty applying a known ratio to find an unknown amount, perhaps particularly if the ratio is unfamiliar.

\section{a. Traxolene question:}

You are part of a team that has invented a high-tech material called "traxolene." Each gram of traxolene has a volume of 2.2 cubic centimeters. For an experiment, you are working with a piece of traxolene that has a volume of $V$ cubic centimeters. To figure out the mass of this piece of traxolene (in grams), you should:
a. divide $V / 2.2$
b. divide $2.2 / \mathrm{V}$
c. multiply $V \bullet 2.2$
d. none of these

b. Olive oil question:

You go to the farmer's market to buy olive oil. When you arrive you realize that you have only one dollar in your pocket. The clerk sells you 0.26 pints of olive oil for one dollar. You plan next week to buy $P$ pints of olive oil. To figure out how much this will cost (in dollars), you should:
a. divide $P / 0.26$
b. divide $0.26 / P$
c. multiply $P \bullet 0.26$
d. none of these

FIG. 1. Questions designed to assess student facility with application of ratio.

\section{CONCLUSIONS}

During a yearlong introductory physics course, students encounter many ratio and product quantities. Lack of facility with ratios may push students toward rote use of formulas, unable to apply proportional reasoning to guide sense making. We are developing a set of natures of proportional reasoning to delineate the specific ways that experts reason with ratios in introductory physics contexts. This paper presents three such natures: verbal interpretation of a ratio, construction of a ratios, and application of a ratio. We expect these will form the core of a more comprehensive set.

Prior work led to development of a validated framework for the natures of negativity in introductory physics. This framework provides a comprehensive road map for the reasoning sub-domain involving the interpretation of signs and signed quantities in introductory physics contexts. Such a map, in turn, serves as a practical guide for the development of assessment questions that can reliably track student progress and evaluate the effectiveness of instruction. We are currently using the framework for the natures of negativity to help us develop a Physics Inventory of Quantitative Literacy (PIQL), which includes assessment questions for student facility with negativity. Our goal is to develop the PIQL into a valid, reliable, and easy to use assessment of mathematization in physics more broadly [17]. A validated framework for proportional reasoning will contribute to this effort.

\section{ACKNOWLEDGMENTS}

The authors gratefully acknowledge the valuable comments of three anonymous reviewers and support from NSF (DUE-1832836, DUE-1833050, DUE-1832880). 
[1] E.F. Redish and E. Kuo, Language of physics, language of math: Disciplinary culture and dynamic epistemology, Science and Education, 24, 561 (2015).

[2] T. Bing and E. F. Redish, Analyzing problem solving using math in physics: Epistemological framing via warrants, Phys. Rev. Phys. Educ. Res., 5, 020108 (2009).

[3] E. Torigoe and G. Gladding, Connecting symbolic difficulties with failure in physics, Am. J. Phys., 79, 133 (2011).

[4] B. Sherin, How students understand physics equations, Cognition and Instruction, 19, 479 (2001).

[5] A. Arons, A Guide to Introductory Physics Teaching (Wiley, New York, 1990).

[6]D. Trowbridge and L. C. McDermott, Investigation of student understanding of the concept of acceleration in one dimension, Am. J. Phys., 48, 242 (1981).

[7] P. Thompson, Quantitative reasoning and mathematical modeling, in New perspectives and directions for collaborative research in mathematics education, edited by L. L. Hatfield, S. Chamberlain, and S. Belbase (WISDOM ${ }^{\mathrm{e}}$ Monographs, University of Wyoming, 2011) pp. 33-57.

[8] S. Brahmia, A. Olsho, T. I. Smith and A. Boudreaux, Framework for the natures of negativity in introductory physics, Phys. Rev. Phys. Educ. Res., 16, 010120 (2020).

[9] E. Gray and D. Tall, Duality, ambiguity and flexibility: A proceptual view of simple arithmetic, J. Res. Math. Educ., 26, 115 (1994).

[10] J. Saldana, The Coding Manual for Qualitative Researchers, (Sage, Los Angeles, 2016), $3^{\text {rd }}$ ed.

[11] http://www.corestandards.org.

[12] J. Larkin, Expert and novice performance in solving physics problems, Science, 208, 1335 (1980).

[13]J. Mestre, R. Dufresne, W. Gerace, and P. Hardiman, Promoting skilled problem-solving behavior among beginning physics students, J. Res. Sci. Teach., 30, 303 (1993).

[14] A. Akatugba and J. Wallace, Mathematical dimensions of students' use of proportional reasoning in high school physics, School Science and Mathematics, 99, 1 (1999).

[15] M. Simon and G. Blume, Mathematical modeling as a component of understand ratio-as-measure: A study of prospective elementary teachers, J. Math. Beh. 13, 183 (1994).

[16] M. A. Simon, Prospective elementary teachers' knowledge of division, J. Res. Math. Educ., 747, 233 (1993).

[17] T. I. Smith, P. Eaton, S.W. Brahmia, A. Olsho, C. Zimmerman and A. Boudreaux, Toward a valid instrument for measuring physics quantitative literacy, in Physics Education Research Conference 2020, PER Conference, edited by Y. Cao, S. Wolf, and M. Bennett (2020). 